\title{
COASSOCIATIVE LIE ALGEBRAS
}

\author{
D.-G. WANG \\ School of Mathematical Sciences, Qufu Normal University, Qufu, Shandong 273165, P. R. China \\ e-mail:dgwang@mail.qfnu.edu.cn \\ J. J. ZHANG \\ Department of Mathematics, Box 354350, University of Washington, Seattle, WA 98195, USA \\ e-mail: zhang@math.washington.edu \\ and G. ZHUANG \\ e-mail: gzhuang@math.washington.edu
}

Dedicated to Kenny Brown and Toby Stafford on the occasion of their 60th birthdays.

\begin{abstract}
A coassociative Lie algebra is a Lie algebra equipped with a coassociative coalgebra structure satisfying a compatibility condition. The enveloping algebra of a coassociative Lie algebra can be viewed as a coalgebraic deformation of the usual universal enveloping algebra of a Lie algebra. This new enveloping algebra provides interesting examples of non-commutative and non-cocommutative Hopf algebras and leads to the classification of connected Hopf algebras of GelfandKirillov dimension four in Wang et al. (Trans. Amer. Math. Soc., to appear).
\end{abstract}

2000 Mathematics Subject Classification. Primary 16A24, 16W30, 57T05.

1. Introduction. We introduce the notion of a coassociative Lie algebra which generalizes in an obvious way both a Lie algebra and a coassociative coalgebra without counit. The enveloping algebra of a coassociative Lie algebra is a bialgebra that is a generalization of the usual universal enveloping algebra of a Lie algebra. The enveloping algebra of a coassociative Lie algebra should be considered as a coalgebraic deformation of the usual universal enveloping algebra on the one hand, and potentially as an algebraic deformation of the co-ordinate ring (regular functions) of certain algebraic groups or semi-groups on the other.

Let $k$ be a base field that is algebraically closed and everything is over $k$.

Let $\mathfrak{g}$ denote an ordinary Lie algebra and $L$ a coassociative Lie algebra. Let $U(\mathfrak{g})$ (respectively, $U(L)$ ) denote the enveloping algebra of $\mathfrak{g}$ (respectively, $L$ ). It is well known that $U(\mathfrak{g})$ is a Hopf algebra. In contrast, $U(L)$ is not a Hopf algebra in general.

THEOREM 1.1 (Theorem 3.5). The enveloping algebra $U(L)$ of a coassociative Lie algebra $L$ is a Hopf algebra if and only if $L$ is locally conilpotent.

Most of the unexplained terms will be defined in Sections 1 and 2.

Starting with conilpotent coalgebras or nilpotnent Lie algebras, one can construct families of nontrivial coassociative Lie algebras based on them. For instance, we give explicit examples in Example 5.4 (based on strictly upper triangular matrix coalgebras) and Example 5.3 (based on Heisenberg Lie algebras). However, it is quite unsatisfactory 
that there is no nontrivial Lie structure based on cosemi-simple coalgebras (Proposition 4.6) and no nontrivial coalgebra structure based on the semi-simple Lie algebra $s l_{2}$ (Theorem 4.9). It would be interesting if a generalized version of coassociative Lie structure can be constructed on cosemi-simple coalgebras and/or semi-simple Lie algebras.

Coassociative Lie algebras are helpful in the classification of connected Hopf algebras of low Gelfand-Kirillov dimension (denoted by GKdim). Definitions and basic properties of GKdim can be found in the first three chapters of [4]. Our reference book for Hopf algebras is [6]. Let $P(H)$ be the subspace of all primitive elements in $H$, and $p(H)$ denote the dimension of $P(H)$. If $\mathrm{GKdim} H \leq 2$, then it is well known that $H \cong U(\mathfrak{g})$ for a Lie algebra $\mathfrak{g}$ of dimension $p(H)$. If $\mathrm{GKdim} H=3$, then it follows from the result of the third author [10] that $H$ is isomorphic to either

(i) $U(\mathfrak{g})$ for a Lie algebra $\mathfrak{g}$ of dimension 3 , or

(ii) $U(L)$ for a coassociative Lie algebra $L$ of dimension 3.

In [7], we give a classification in GKdim 4.

THEOREM 1.2. [7, Theorem 0.3 and Remark 0.4]. Suppose that $k$ is of characteristic zero. Let $H$ be a connected Hopf algebra of GKdim 4. Then one of the following occurs:

(a) If $p(H)=4$, then $H \cong U(\mathfrak{g})$ for a Lie algebra $\mathfrak{g}$ of dimension 4 .

(b) If $p(H)=3$, then $H \cong U(L)$ for an anti-cocommutative coassociative Lie algebra $L$ of dimension 4.

(c) If $p(H)=2$, then $H$ is not isomorphic to either $U(\mathfrak{g})$ or $U(L)$ as Hopf algebras, for any Lie algebra $\mathfrak{g}$ or any coassociative Lie algebra L. Such a Hopf algebra is isomorphic to one of the four families of Hopf algebras explicitly constructed in [7, Section 4].

The Hopf algebras in Theorem 1.2(b) and (c) are completely classified in [7]. The proof of Theorem 1.2 is heavily dependent on the study of coassociative Lie algebras. A result of the Milnor-Moore-Cartier-Kostant Theorem [6, Theorem 5.6.5] states that any cocommutative connected Hopf algebra over a field of characteristic zero is isomorphic to $U(\mathfrak{g})$ for some Lie algebra $\mathfrak{g}$. This applies to case (a) of Theorem 1.2. However, the Hopf algebras in Theorem 1.2(b) and (c) are not cocommutative, hence not isomorphic to $U(\mathfrak{g})$ for a usual Lie algebra $\mathfrak{g}$. A generalization of Theorem 1.2(b) states that if $p(H)=\mathrm{GKdim} H-1$, then $H$ is isomorphic to $U(L)$ for some coassociative Lie algebra $L[7$, Theorem 0.5$]$.

The Hopf algebra $U(\mathfrak{g})$ is always involutory. In general, $U(L)$ is not involutory. By using the Calabi-Yau property of the enveloping algebra of unimodular Lie algebras, we prove the following result.

THEOREM 1.3 (Theorem 3.7). Let $\mathfrak{g}$ be a finite dimensional unimodular Lie algebra. Suppose that $L:=(\mathfrak{g}, \delta)$ is a conilpotent coassociative Lie algebra. Then $U(L)$ is involutory.

Coassociative Lie algebras are not understood fully, and a lot of basic questions remain unsolved. For example, the following:

QUESTION 1.4. Let $n(L)$ be the nilpotency of $L$ and $\operatorname{con}(L)$ be the conilpotency of $L$. If $n(L)+\operatorname{con}(L)<\infty$, then is there the bound for the number $n(L)+\operatorname{con}(L)-\operatorname{dim} L$ ?

Preliminary analysis of known examples shows that if $n(L)+\operatorname{con}(L)<\infty$, then

$$
n(L)+\operatorname{con}(L)-\operatorname{dim} L \leq 1 .
$$


As one may guess, this paper grows out of the study of connected Hopf algebras [7]. By Theorem 1.2(b), certain classes of connected Hopf algebras are the enveloping algebras of coassociative Lie algebras, which are non-commutative and non-cocommutative. This construction is very different from the various classical constructions of quantum groups which we are familiar with. One way of thinking of a coassociative Lie algebra is that it has an additional coalgebra structure on a Lie algebra. Our work [7] suggests that certain 'nilpotent' quantum groups could be represented by a Lie algebra with an extra coalgebra structure. This idea might be worth pursuing further.

2. Definitions. In this section, we start with some definitions and give some easy examples of coassociative Lie algebras.

Definition 2.1. A Lie algebra $(L,[]$,$) together with a coproduct \delta: L \rightarrow L \otimes L$ is called a coassociative Lie algebra if

(a) $(L, \delta)$ is a coassociative coalgebra without counit, and

(b) two operations $\delta$ and [, ] satisfy the following compatibility condition

$$
\delta([a, b])=b_{1} \otimes\left[a, b_{2}\right]+\left[a, b_{1}\right] \otimes b_{2}+\left[a_{1}, b\right] \otimes a_{2}+a_{1} \otimes\left[a_{2}, b\right]+[\delta(a), \delta(b)]
$$

for all $a, b \in L$.

Here $\delta(x)=x_{1} \otimes x_{2}$, which is basically Sweedler's notation with the summation indicator omitted.

Definition 2.1 is not complete without the following remark.

REMARK 2.2. For a general Lie algebra $L$, the product $L \otimes L$ is not a Lie algebra in any natural way. To make sense of $[\delta(a), \delta(b)]$ in (E2.1.1), we need to embed $L$ into the usual universal enveloping algebra $U(L)$. Then $L \otimes L$ can be naturally identified with a subspace of $U(L) \otimes U(L)$. Under this identification, $[f, g]$ is defined as the commutator of $f$ and $g$ in the associative algebra $U(L) \otimes U(L)$ for all elements $f, g \in$ $L \otimes L$. Equation (E2.1.1) implies that the element $[\delta(a), \delta(b)] \in U(L) \otimes U(L)$ is actually in the subspace $L \otimes L$, namely

$$
[\delta(a), \delta(b)] \in L \otimes L
$$

Inside $U(L) \otimes U(L)$, equation (E2.1.1) can be written as

$$
\delta([a, b])=[a \otimes 1+1 \otimes a, \delta(b)]+[\delta(a), b \otimes 1+1 \otimes b]+[\delta(a), \delta(b)]
$$

for all $a, b \in L$. Let $L^{1}$ denote the Lie algebra extension $L \oplus k 1$, where 1 is in the centre (i.e. $\left.\left[1, L^{1}\right]=0\right)$. Define $\Delta: L^{1} \rightarrow L^{1} \otimes L^{1}$ by

$$
\Delta(1)=1 \otimes 1, \quad \Delta(a)=a \otimes 1+1 \otimes a+\delta(a)
$$

for all $a \in L$. By embedding $L^{1}=L \oplus k 1$ into $U(L)$ naturally, we can define $[f, g]$ for all elements in $f, g \in L^{1}$. For any $k$-space $V$, let $V^{\otimes 2}$ denote $V \otimes V$.

LemMA 2.3. Let $L$ be a Lie algebra, and let $\delta: L \rightarrow L^{\otimes 2}$ be any linear map. Then $L$ is a coassociative Lie algebra if and only if $\left(L^{1}, \Delta\right)$ is a counital coalgebra and the 
equation

$$
\Delta([a, b])=[\Delta(a), \Delta(b)]
$$

holds in $U(L)^{\otimes 2}$ for all $a, b \in L^{1}$.

Proof. A direct calculation shows that $\Delta$ is coassociative if and only if $\delta$ is coassociative. It is also easy to see that (E2.1.1) is equivalent to (E2.3.1).

It follows from $(\mathrm{E} 2.2 .2)$ that the kernel $\operatorname{ker} \delta$ is a Lie subalgebra of $L$. Let

$$
\Phi(a, b):=\delta([a, b])-\left(b_{1} \otimes\left[a, b_{2}\right]+\left[a, b_{1}\right] \otimes b_{2}+\left[a_{1}, b\right] \otimes a_{2}+a_{1} \otimes\left[a_{2}, b\right]\right) .
$$

Then (E2.1.1) becomes

$$
\Phi(a, b)=[\delta(a), \delta(b)] .
$$

Let $L$ be a coassociative Lie algebra. If $\delta=0$, it is an ordinary Lie algebra. If [ , ] = 0 , then $L$ is simply a coassociative coalgebra without counit. Let CoLieAlg denote the category of coassociative Lie algebras, LieAlg denote the category of Lie algebras and CoAlg denote the category of coalgebras without counit. Then both LieAlg and CoAlg are full subcategories of CoLieAlg. Now we give some simple examples of coassociative Lie algebras with nontrivial Lie bracket [, ] and nontrivial coproduct $\delta$, in which both sides of (E2.1.1) are trivially zero. More complicated examples will be given in the later sections.

EXAMPLE 2.4. Let $L=k x \oplus k y$ with $[x, y]=y$ and $\delta(x)=\lambda y \otimes y$ for any $\lambda \in k$ and $\delta(y)=0$. Then $L$ is a coassociative Lie algebra. It is clear that $(L,[]$,$) is a Lie$ algebra and $(L, \delta)$ is a coalgebra. Note that (E2.1.1) is trivial when $a=b$. So it suffices to check (E2.1.1) for $a=x$ and $b=y$, in which case both sides of (E2.1.1) are zero. It can be verified that, up to isomorphism, this is the unique coassociative Lie algebra with an underlying non-abelian Lie algebra of dimension 2.

Here is a slightly different situation.

LeMma 2.5. Let $(L,[]$,$) be a Lie algebra and (L, \delta)$ be a coalgebra. Suppose that $\delta(L) \subset Z \otimes Z$, where $Z$ is the centre of $(L,[]$,$) and that \delta([L, L])=0$. Then $L$ is $a$ coassociative Lie algebra.

Proof. The hypotheses imply that both sides of (E2.1.1) are zero.

EXAMPLE 2.6. Suppose that $L$ is a Lie algebra containing a Lie ideal $W$ such that both $W$ and $L / W$ are abelian. For example, $L$ is a Lie algebra of nilpotency 2 . Let $\delta: L \stackrel{\pi}{\rightarrow} L / W \rightarrow W^{\otimes 2}$ be a $k$-linear map where $\pi$ is the quotient map. A direct calculation shows that $(\delta \otimes 1) \delta=(1 \otimes \delta) \delta=0$. Thus, $(L, \delta)$ is coassociative. By the definition of $\delta$, the hypotheses of Lemma 2.5 hold; whence $L$ is a coassociative Lie algebra. Below are two special cases.

(a) Let $L$ be the 3-dimensional Heisenberg Lie algebra with a basis $\{x, y, z\}$ such that $[x, y]=z$ and $z$ is central. Define $\delta(x)=z \otimes z$ and $\delta(y)=\lambda z \otimes z$, for some $\lambda \in k$, and $\delta(z)=0$. By the above paragraph, $L$ is a coassociative Lie algebra.

(b) Let $W_{1}$ be any Lie algebra and $W$ be a vector space. Let $\phi: W_{1} \rightarrow W$ be any $k$-linear map. Define a Lie bracket on $L:=W_{1} \oplus W$ by

$$
\left[w_{1}+w, t_{1}+t\right]=\phi\left(\left[w_{1}, t_{1}\right]_{W_{1}}\right)
$$


for all $w_{1}, t_{1} \in W_{1}$ and $w, t \in W$. Then $W$ is a Lie ideal of $L$ such that both $L / W$ and $W$ are abelian. Then every $k$-linear map $\delta: L \stackrel{\pi}{\rightarrow} W_{1} \rightarrow W \otimes W$ defines a coassociative Lie algebra $L$.

Next, we give an example of 'almost coassociative Lie algebra', which is dependent on the embedding of $L$ into an associative algebra. If $A$ is any associative algebra, define $[,]_{A}$ to be the commutator of $A$.

EXAMPLE 2.7. Let $H$ be a bialgebra and let $H_{+}:=\operatorname{ker} \epsilon$, where $\epsilon$ is the counit of $H$. Let $L$ be a Lie subalgebra of $\left(H_{+},[,]_{H}\right)$ (for example, $\left.L=H_{+}\right)$. Define $\delta(a)=\Delta(a)-$ $1 \otimes a-a \otimes 1$ for all $a \in H$. Then $\left(H_{+}, \delta\right)$ is a coalgebra. Suppose that $\delta(L) \subset L^{\otimes 2}$. Then $(L, \delta)$ is a coalgebra. However, $\left(L,[,]_{H}, \delta\right)$ is generally not a coassociative Lie algebra.

Let $H$ be the 4-dimensional Taft Hopf algebra:

$$
k\langle g, x\rangle /\left(x g+g x=0, g^{2}=1, x^{2}=0\right)
$$

with $\Delta(g)=g \otimes g, \Delta(x)=x \otimes 1+g \otimes x$, and $\epsilon(g)=1, \epsilon(x)=0$. Let $L$ be $H_{+}=k x \oplus$ $k(g-1) \oplus k(g x)$. Let $y=g-1$ and $z=g x$. Then $[x, y]_{H}=-2 z,[x, z]_{H}=0,[y, z]_{H}=$ $2 x, \delta(x)=y \otimes x, \delta(y)=y \otimes y$, and $\delta(z)=z \otimes y$. Hence,

$$
[\delta(x), \delta(z)]_{H^{\otimes 2}}=[y \otimes x, z \otimes y]_{H^{\otimes 2}}=y z \otimes x y-z y \otimes y x=:(*) .
$$

Using the facts $[x, y]_{H}=-2 z$ and $[y, z]_{H}=2 x$, we have

$$
(*)=y z \otimes x y-(y z-2 x) \otimes(x y+2 z)=2 y z \otimes z+2 x \otimes x y+4 x \otimes z,
$$

which is not in $L \otimes L$ if we embed $L$ into $U(L)$. Hence, (E2.1.1) does not hold for $\left(L,[,]_{H}, \delta\right)$, and consequently $L$ is not a coassociative Lie algebra. After identifying $y z$ with $-z$, and $x y$ with $-x-z$ in $H,($ E2.1.1) does hold in $H \otimes H$.

The enveloping algebra of a coassociative Lie algebra is defined as follows.

DEFINITION 2.8. Let $L$ be a coassociative Lie algebra. The enveloping algebra of $L$, denoted by $U(L)$, is defined to be a bialgebra, whose algebra structure equals that of the enveloping algebra of the underlying Lie algebra $L$, namely,

$$
U(L)=k\langle L\rangle /(a b-b a=[a, b], \forall a, b \in L),
$$

and whose coalgebra structure is determined by

$$
\Delta(a)=a \otimes 1+1 \otimes a+\delta(a), \quad \epsilon(a)=0
$$

for all $a \in L$. By (E2.3.1) it is easy to see that $U(L)$ is a bialgebra. We will also use $U(L, \delta)$ to denote $U(L)$ if we want to emphasize the coproduct $\delta$.

It is clear that the assignment $L \rightarrow U(L)$ defines a functor from CoLieAlg to BiAlg, where BiAlg is the category of bialgebras.

EXAMPLE 2.9. If $\operatorname{dim} L=1$, then there are exactly two coassociative Lie algebra structures on $L$ up to isomorphism. One is determined by $\delta=0$. In this case the enveloping algebra is $U(L)=k[x]$ with $x$ being a primitive element. Consequently, $U(L)$ is a Hopf algebra. The other is determined by $\delta(x)=x \otimes x$. Here $U(L)=k[g]$, 
where $g=1+x$ and $g$ is a group-like element in $U(L)$. In this case $U(L)$ is not a Hopf algebra because the group-like element $g$ is not invertible in $U(L)$.

Let $(\mathfrak{g}, \delta)$ be a coassociative Lie algebra with underlying Lie algebra $\mathfrak{g}$. Then the Poincaré-Birkhoff-Witt (PBW) theorem holds for $U(\mathfrak{g}, \delta)$, since algebraically it is the usual enveloping algebra $U(\mathfrak{g})$. The difference between $U(\mathfrak{g}, \delta)$ and $U(\mathfrak{g})$ is their coalgebra structures. For many examples of $(\mathfrak{g}, \delta)$, one can construct explicitly a family of bialgebras $B(q)$ dependent on $(\mathfrak{g}, \delta)$, where $q \in k$, such that $B(1)=U(\mathfrak{g}, \delta)$ and $B(0)=U(\mathfrak{g})$. Hence, $U(\mathfrak{g}, \delta)$ can be considered as a coalgebraic deformation of $U(\mathfrak{g})$. However, we will not pursue this topic further.

Since $U(L)$ is generated by $L$ as an algebra, $U(L)$ is cocommutative if and only if the underlying coalgebra $L$ is cocommutative. Similarly, $U(L)$ is commutative if and only if the underlying Lie algebra $L$ is abelian.

Let $\delta^{n}=\left(\delta \otimes 1^{\otimes n-1}\right)\left(\delta \otimes 1^{\otimes n-2}\right) \cdots(\delta \otimes 1) \delta$. Here is a list of definitions.

DeFINITION 2.10. Let $L_{1}, L_{2}, L$ be coassociative Lie algebras.

(a) We say that $L_{1}$ and $L_{2}$ are quasi-equivalent if $U\left(L_{1}\right)$ is isomorphic to $U\left(L_{2}\right)$ as bialgebras.

(b) A Lie algebra $\mathfrak{g}$ is called rigid if every compatible $\delta$-structure on $\mathfrak{g}$ is zero.

(c) A coalgebra $C$ is called rigid if every compatible Lie-structure on $C$ is trivial.

(d) A coalgebra $(C, \delta)$ is called anti-cocommutative if $\tau \delta=-\delta$, where the flip $\tau: C^{\otimes 2} \rightarrow C^{\otimes 2}$ is defined by $\tau(a \otimes b)=b \otimes a$.

(e) The nilpotency of $L$, denoted by $n(L)$, is defined to be the nilpotency of the underlying Lie algebra $L$.

(f) An element $x \in L$ is called conilpotent if $\delta^{n}(x)=0$ for some $n>0$. We say $L$ is locally conilpotent if every element in $L$ is conilpotent.

(g) We call $L n$-conilpotent if $\delta^{n}(L)=0$. The smallest such $n$, denoted by $\operatorname{con}(L)$, is called conilpotency of $L$.

3. Results on enveloping algebras. In this section we study some properties of the enveloping algebras $U(L)$. Let $B$ be a bialgebra with coproduct $\Delta$. Define $\delta_{B}: B \rightarrow B^{\otimes 2}$ by

$$
\delta_{B}(x)=\Delta(x)-x \otimes 1-1 \otimes x
$$

for all $x \in B$.

Definition 3.1. A subspace $V$ in a bialgebra $B$ is called a $\delta$-space of $B$ if

(a) $V$ is a Lie subalgebra of $\left(B,[,]_{B}\right)$,

(b) $\epsilon(V)=0$,

(c) $\delta_{B}(V) \subset V^{\otimes 2}$ inside $B^{\otimes 2}$ and

(d) $B$ is an $\mathbb{N}$-filtered algebra with an exhaustive filtration defined by $F_{n}(B):=$ $(k 1+V)^{n}$, for $n \geq 0$, such that the associated graded $\operatorname{ring} g r_{F} B$ is isomorphic to the commutative polynomial ring $k[V]$.

REMARK 3.2. If $L$ is coassociative Lie algebra, then $L$ is $\delta$-space of $U(L)$. In general, a $\delta$-space of $U(L)$ is not unique. See Corollary 3.6.

LEMMA 3.3. If $V$ is a $\delta$-space of $B$, then $\left(V,[,]_{B}, \delta_{B}\right)$ is a coassociative Lie algebra and $B \cong U(V)$ as bialgebras. 
Proof. Let $U$ be the usual enveloping algebra of the Lie algebra $\left(V,[,]_{B}\right)$. Then there is an algebra homomorphism $\phi: U \rightarrow B$ such that $\left.\phi\right|_{V}=I d_{V}$. It follows from Definition 3.1(c) that $B$ is generated by $V$ and that the set $\left\{v_{1}^{n_{1}} \cdots v_{d}^{n_{d}} \mid n_{i} \geq 0\right\}$ is a $k$ linear basis of $B$ where $\left\{v_{1}, \cdots, v_{j}, \cdots\right\}$ is a $k$-linear basis of $V$. Since $\left\{v_{1}^{n_{1}} \cdots v_{d}^{n_{d}} \mid n_{i} \geq\right.$ $0\}$ is also a $k$-linear basis of $U$ by the PBW theorem, $\phi$ is an isomorphism of algebras. Note that $B$ is a bialgebra and generated by $V$ as an algebra, one can define a canonical bialgebra structure $\Delta_{U}$ on $U$ via $\phi$ such that $\phi$ is an isomorphism of bialgebras. Let $\delta_{U}(v)=\Delta_{U}(v)-v \otimes 1-1 \otimes v$ for $v \in V$. Since $\delta_{B}(x)=\Delta_{B}(x)-x \otimes 1-1 \otimes x$, $\delta_{U}(v)=\delta_{B}(v)$ for all $v \in V$ (we are identifying the subspace $V \subset B$ with the subspace $V \subset U$ via the map $\left.\left.\phi\right|_{V}=I d_{V}\right)$. By Definition 3.1(b), one sees easily that $\delta_{U}(v) \in V^{\otimes 2}$ for all $v \in V$. Since $U$ is a bialgebra (via the map $\phi),($ E2.3.1) holds. Now by Lemma 2.3 and Definition 2.8, $\left(V,[,]_{U}, \delta_{U}\right)$ is a coassociative Lie algebra with enveloping algebra $U$. Since $\left(V,[,]_{B}, \delta_{B}\right)=\left(V,[,]_{U}, \delta_{U}\right)$ by construction and $U \cong B$ as bialgebras, the results follow.

Let $(L, \delta)$ be a coalgebra (without counit). Let $L^{1}=k 1 \oplus L$, and $\Delta: L^{1} \rightarrow\left(L^{1}\right)^{\otimes 2}$ be defined as (E2.2.3). Moreover, let $\epsilon: L^{1} \rightarrow k$ be defined by $\epsilon(1)=1, \epsilon(x)=0$ for all $x \in L$. Then the assignment $(L, \delta) \rightarrow\left(L^{1}, \Delta, \epsilon\right)$ defines a functor from CoAlg to CouAlg, where CouAlg is the category of counital coassociative coalgebras. The following lemma is easy.

Lemma 3.4. Let $(L, \delta)$ be a coalgebra. Then $(L, \delta)$ is locally conilpotent if and only if $\left(L^{1}, \Delta, \epsilon\right)$ is a connected counital coalgebra.

Proof. Since $L$ is a sum of its finite dimensional subcoalgebras, we can assume without loss of generality that $L$ is finite dimensional. Note that $L$ can be identified with the quotient coalgebra $L^{1} / k 1$. By taking the $k$-linear dual, $L^{*}$ becomes a subalgebra (without a unit) of $\left(L^{1}\right)^{*}$. In fact, $L^{*}$ is a maximal ideal of $\left(L^{1}\right)^{*}$ of co-dimension 1. Now the lemma is equivalent to the statement that $L^{*}$ is a nilpotent ideal if and only if $L^{*}$ is the unique maximal ideal of $\left(L^{1}\right)^{*}$, which is an easy ring-theoretical fact.

Now we are ready to prove Theorem 1.1.

THEOREM 3.5. Let L be a coassociative Lie algebra. Then the following are equivalent:

(a) L is locally conilpotent;

(b) $U(L)$ is a connected Hopf algebra and

(c) $U(L)$ is a Hopf algebra.

Proof. (a) $\Rightarrow$ (b). Since $(L, \delta)$ is locally conilpotent, $\left(L^{1}, \Delta, \epsilon\right)$ is a connected coalgebra by Lemma 3.4. Since $U(L)$ is generated by $L^{1}$ as an algebra, $U(L)$ is connected as a coalgebra. It follows from [6, Lemma 5.2.10] that a connected bialgebra is automatically a Hopf algebra.

(b) $\Rightarrow$ (c). This is clear.

(c) $\Rightarrow$ (a). We proceed by contradiction. Suppose that $U(L)$ is a Hopf algebra, but $(L, \delta)$ is not locally conilpotent. Then $\left(L^{1}, \Delta\right)$ is not connected, whence its co-radical is strictly larger than $k 1$. Pick a simple subcoalgebra with counit of $L^{1}$, say $C$, which is not equal to $k 1$. Since $k$ is algebraically closed, $C$ is isomorphic to a matrix coalgebra $\bigoplus_{i, j=1, \ldots, n} k x_{i j}$ with

$$
\Delta\left(x_{i j}\right)=\sum_{s=1}^{n} x_{i s} \otimes x_{s j}, \quad \text { and } \quad \epsilon\left(x_{i j}\right)=\delta_{i j}
$$


for all $1 \leq i, j \leq n$. Here $\delta_{i j}$ is the Kronecker delta. Let $y_{i j}=x_{i j}-\delta_{i j}$, for all $i, j$. Note that $L=\operatorname{ker}\left(\epsilon: L^{1} \rightarrow k\right)$. Then $\bigoplus_{i, j=1, \ldots, n} k y_{i j} \subset L$ is a simple subcoalgebra of $L$ such that $\delta\left(y_{i j}\right)=\sum_{s=1}^{n} y_{i s} \otimes y_{s j}$ for all $1 \leq i, j \leq n$. Let $z_{i j}=S\left(x_{i j}\right)$ for all $1 \leq i, j \leq n$. Let $X$ be the matrix $\left(x_{i j}\right)_{n \times n}$ and $Z$ be the matrix $\left(z_{i j}\right)_{n \times n}$. Then the antipode axiom implies that $X Z=Z X=I_{n}$, where $I_{n}$ is the identity $n \times n$-matrix. Note that $L$ is a $\delta$-space of $U(L)$, and hence $U(L)$ has a filtration defined by $F_{n}=\left(L^{1}\right)^{n}$ such that $\operatorname{gr}_{F} U(L)$ is isomorphic to the commutative polynomial ring $k[L]$. One can extend this filtration naturally from $U(L)$ to the matrix algebra $M_{n}(U(L))$ such that $\operatorname{gr}_{F}\left(M_{n}(U(L)) \cong M_{n}(k[L])\right.$. Let gr also denote the leading terms of elements in $\operatorname{gr}_{F}\left(M_{n}(U(L))\right.$. Then the equation $X Z=I_{n}$ implies that $\operatorname{gr}(X) \operatorname{gr}(Z)=0$. Note that $\operatorname{gr}(X)=\left(y_{i j}\right) \in M_{n}(k[L])$ and thus $\operatorname{det} \operatorname{gr}(X)=\operatorname{det}\left(y_{i j}\right)$, which is non-zero in the commutative polynomial subring $k\left[y_{i j}\right] \subset$ $k[L]$. Consequently, equation $\operatorname{gr}(X) \operatorname{gr}(Z)=0$ implies that $\operatorname{gr}(Z)=0$. Hence, $Z=0$, yielding a contradiction. The assertion follows.

COROLlARY 3.6. If char $k=0$, then every locally conilpotent cocommutative coassociative Lie algebra is quasi-equivalent to a Lie algebra.

Proof. Let $L$ be any locally conilpotent cocommutative coassociative Lie algebra. Then $U(L)$ is a connected cocommutative Hopf algebra by Theorem 3.5. By the Milnor-Moore-Cartier-Kostant Theorem [6, Theorem 5.6.5], $U(L)$ is isomorphic to $U(\mathfrak{g})$ for some Lie algebra $\mathfrak{g}$. The assertion follows.

Recall that a Lie algebra $\mathfrak{g}$ is called unimodular if $\operatorname{ad}(x)$ has zero trace for all $x \in \mathfrak{g}$, where $a d(x) \in \operatorname{End}_{k}(\mathfrak{g})$ is the $k$-linear map sending $y \in \mathfrak{g}$ to $[x, y]$. Combining results of Koszul [3] and Yekutieli [8, Theorem A] (also see [1, Proposition 6.3] and [2, Theorem 5.3 and Lemma 4.1]), $\mathfrak{g}$ is unimodular if and only if $U(\mathfrak{g})[d]$ is the rigid dualizing complex over $U(\mathfrak{g})$ if $d:=\operatorname{dim} \mathfrak{g}$ is finite. By [1, Proposition 6.3] and [2, Theorem 5.3], $\mathfrak{g}$ is unimodular if and only if the Hopf algebra $U(\mathfrak{g})$ is unimodular in the sense of [5], if and only if $U(\mathfrak{g})$ is Calabi-Yau and if and only if the homological integral of $U(\mathfrak{g})$ (as defined in [5]) is trivial. It is well known that all Heisenberg Lie algebras are unimodular, and that the 2-dimensional non-abelian Lie algebra is not. Next we verify Theorem 1.3.

THEOREM 3.7. Let $\mathfrak{g}$ be a finite-dimensional unimodular Lie algebra. Suppose $(\mathfrak{g}, \delta)$ is a coassociative Lie algebra such that $\delta$ is conilpotent. Then the Hopf algebra $U(\mathfrak{g}, \delta)$ is involutory.

Proof. Let $H$ (respectively $K$ ) denote the Hopf algebra $U(\mathfrak{g}, \delta)$ (respectively, $U(\mathfrak{g})$ ). Let $\mu_{H}$ and $\mu_{K}$ denote the Nakayama automorphisms of $H$ and $K$ respectively. Since the Nakayama automorphism is defined uniquely up to an inner automorphism, and the units in $H$ are those elements in $k^{\times}$, the Nakayama automorphism of $H$ (and of $K$ ) is unique. Since $H=K$ as algebras by Definition 2.8, we have $\mu_{H}=\mu_{K}$. Since $\mathfrak{g}$ is unimodular, $\mu_{K}=I d_{K}$ by [1, Proposition 6.3(c)]. As a consequence $\mu_{H}=I d_{H}$.

Since $\mathfrak{g}$ is unimodular, the homological integral of $K$, denoted by $\int_{K}^{l}$, is trivial. Consequently, $\int_{K}^{l}$ equals the trivial module $K /(\mathfrak{g})$, where $(\mathfrak{g})$ is the ideal of $K$ generated by subspace $\mathfrak{g}$. The homological integral is only dependent on the algebra structure of the Hopf algebra, so we have $\int_{H}^{l}=H /(\mathfrak{g})$. This implies that $\int_{H}^{l}$ is trivial. Therefore, the left winding automorphism associated to $\int_{H}^{l}$, denoted by $\Xi_{\int^{l}}^{l}$, is the identity map of $H$. 
Combining the above with [1, Theorem 0.3], we have

$$
I d_{H}=\mu_{H}=S_{H}^{2} \circ \Xi_{f^{l}}^{l}=S_{H}^{2} \circ I d_{H}=S_{H}^{2},
$$

where $S_{H}$ is the antipode of $H$. Hence, $S_{H}^{2}=I d_{H}$, and $H$ is involutory.

Example 5.2 shows that $U(\mathfrak{g}, \delta)$ may not be involutory if $\mathfrak{g}$ is not unimodular. This is another way of showing that $U(\mathfrak{g}, \delta)$ is not isomorphic to $U\left(\mathfrak{g}^{\prime}\right)$ for any Lie algebra $\mathfrak{g}^{\prime}$. For the rest of this section we assume that char $k \neq 2$.

LEMMA 3.8. Let $L$ be an anti-cocommutative coalgebra. Then

(a) $\operatorname{con}(L) \leq 2$, and as a consequence, $L$ is conilpotent; and

(b) $\delta(L) \subset(\operatorname{ker} \delta)^{\otimes 2}$.

Proof. (a) A standard calculation by using Sweedler's notation shows that

$$
(1 \otimes \delta) \tau \delta=(\tau \otimes 1)(1 \otimes \tau)(1 \otimes \delta) \delta .
$$

Since $\tau \delta=-\delta$ by assumption, the left-hand side of the equation becomes $-(1 \otimes \delta) \delta$, while the right-hand side is $(1 \otimes \delta) \delta$. As a consequence, $(1 \otimes \delta) \delta=0$.

(b) For any $x \in L$, write $\delta(x)=\sum_{i=1}^{n} x_{i} \otimes y_{i}$ for a minimal integer $n$. Then $\left\{x_{i}\right\}_{i=1}^{n}$ is linearly independent. Since $\operatorname{con}(L) \leq 2$,

$$
0=(1 \otimes \delta) \delta(x)=\sum_{i=1}^{n} x_{i} \otimes \delta\left(y_{i}\right)
$$

Since $\left\{x_{i}\right\}_{i=1}^{n}$ is linearly independent, $\delta\left(y_{i}\right)=0$ for all $i$. This means that $\delta(L) \subset L \otimes$ $\operatorname{ker} \delta$. Similarly, $\delta(L) \subset \operatorname{ker} \delta \otimes L$. The assertion follows.

Lemma 3.8 also implies a nice fact about the form of the antipode.

Proposition 3.9. Suppose that $L$ is anti-cocommutative and $U(L)$ is involutory. Then $S(x)=-x$ for all $x \in L$.

Proof. It follows from Definition 2.1 that the kernel of $\delta$, denoted by $K$, is a Lie subalgebra of $L$. If $x \in K$, then $\Delta(x)=x \otimes 1+1 \otimes x$. The antipode axiom implies that $S(x)=-x$. If $x \in L \backslash K$, it follows from Lemma 3.8(b) that

$$
\delta(x)=\sum_{i<j} a_{i j}\left(x_{i} \otimes x_{j}-x_{j} \otimes x_{i}\right)
$$

for some $x_{i} \in K$ and some $a_{i j} \in k$. Hence,

$$
\Delta(x)=x \otimes 1+1 \otimes x+\sum_{i<j} a_{i j}\left(x_{i} \otimes x_{j}-x_{j} \otimes x_{i}\right) .
$$

Applying the antipode axiom, and using the fact that $S\left(x_{i}\right)=-x_{i}$, we have that

$$
0=S(x)+x+\sum_{i<j} a_{i j}\left(S\left(x_{i}\right) x_{j}-S\left(x_{j}\right) x_{i}\right)=S(x)+x+\sum_{i<j} a_{i j}\left(-x_{i} x_{j}+x_{j} x_{i}\right) .
$$

So $S(x)=-x-Y$, where $Y=\sum_{i<j} a_{i j}\left(-x_{i} x_{j}+x_{j} x_{i}\right) \in K$. Applying $S$ to $S(x)=-x-$ $Y$, and using the hypothesis that $S^{2}=I d$, we have $x=-S(x)+Y$. Thus, $Y=0$, and $S(x)=-x$. 
4. Elementary properties of coassociative Lie algebras. In this section some elementary properties of coassociative Lie algebras are discussed. First we need some lemmas that will simplify computations.

LEMMA 4.1. Let $L$ be a coassociative Lie algebra. Let $\left\{x_{i}\right\}_{i \in I}$ be a totally ordered $k$-linear basis of $L$. For any $a, b \in L$, write $\delta(a)=\sum_{i} x_{i} \otimes a_{i}$ and $\delta(b)=\sum_{i} x_{i} \otimes b_{i}$. Then

(a) $\left[a_{i}, b_{i}\right]=0$ for all $i$; and

(b) $\left[a_{i}, b_{j}\right]+\left[a_{j}, b_{i}\right]=0$ for all $i, j$.

If $\delta(L) \subset L^{\prime} \otimes L^{\prime \prime}$ for some subspaces $L^{\prime}$ and $L^{\prime \prime}$ of $L$, then

(c) $[\delta(a), \delta(b)] \in\left[L^{\prime}, L^{\prime}\right] \otimes\left[L^{\prime \prime}, L^{\prime \prime}\right]$;

(d) $\Phi(a, b) \in\left[L^{\prime}, L^{\prime}\right] \otimes\left[L^{\prime \prime}, L^{\prime \prime}\right]$ and

(e) $\delta([L, L]) \subset\left[L^{\prime}, L\right] \otimes L^{\prime \prime}+L^{\prime} \otimes\left[L^{\prime \prime}, L\right]+\left[L^{\prime}, L^{\prime}\right] \otimes\left[L^{\prime \prime}, L^{\prime \prime}\right]$.

Proof. We compute $[\delta(a), \delta(b)]$ in $U(L)^{\otimes 2}$ as follows:

$$
\begin{aligned}
{[\delta(a), \delta(b)]=} & \sum_{i, j} x_{i} x_{j} \otimes a_{i} b_{j}-\sum_{i, j} x_{i} x_{j} \otimes b_{i} a_{j} \\
= & \sum_{i<j} x_{i} x_{j} \otimes a_{i} b_{j}+\sum_{j>i} x_{j} x_{i} \otimes a_{j} b_{i}+\sum_{i} x_{i}^{2} \otimes a_{i} b_{i} \\
& -\sum_{i<j} x_{i} x_{j} \otimes b_{i} a_{j}-\sum_{j>i} x_{j} x_{i} \otimes b_{j} a_{i}-\sum_{i} x_{i}^{2} \otimes b_{i} a_{i} \\
= & \sum_{i<j} x_{i} x_{j} \otimes\left(a_{i} b_{j}-b_{i} a_{j}\right)+\sum_{i} x_{i}^{2} \otimes\left[a_{i}, b_{i}\right] \\
& +\sum_{j>i}\left(x_{i} x_{j}+\left[x_{j}, x_{i}\right]\right) \otimes a_{j} b_{i}-\sum_{j>i}\left(x_{i} x_{j}+\left[x_{j}, x_{i}\right]\right) \otimes b_{j} a_{i} \\
= & \sum_{i<j} x_{i} x_{j} \otimes\left(\left[a_{i}, b_{j}\right]+\left[a_{j}, b_{i}\right]\right)+\sum_{i} x_{i}^{2} \otimes\left[a_{i}, b_{i}\right] \\
& +\sum_{i<j}\left[x_{i}, x_{j}\right] \otimes\left(b_{j} a_{i}-a_{j} b_{i}\right) .
\end{aligned}
$$

Since $\left\{x_{i} x_{j}\right\}_{i \leq j}$ are linearly independent in $U(L) / L$ and $\left[x_{i}, x_{j}\right] \in L$ for all $i, j$, we have $\left[a_{i}, b_{j}\right]+\left[a_{j}, b_{i}\right]=0,\left[a_{i}, b_{i}\right]=0$. Parts (a) and (b) follow.

Now assume that $\delta(L) \subset L^{\prime} \otimes L^{\prime \prime}$. By the above computation and parts (a) and (b), $[\delta(a), \delta(b)]=\sum_{i<j}\left[x_{i}, x_{j}\right] \otimes\left(b_{j} a_{i}-a_{j} b_{i}\right)$, which is in $\left[L^{\prime}, L^{\prime}\right] \otimes U(L)$, as we can assume $x_{i} \in L^{\prime}$ whenever $a_{i}$ (or $b_{i}$ ) is non-zero. By symmetry, $[\delta(a), \delta(b)] \in U(L) \otimes\left[L^{\prime \prime}, L^{\prime \prime}\right]$. Hence, $[\delta(a), \delta(b)] \in\left[L^{\prime}, L^{\prime}\right] \otimes\left[L^{\prime \prime}, L^{\prime \prime}\right]$. This is part (c). Part (d) follows from the equation $\Phi(a, b)=[\delta(a), \delta(b)]$.

Part (e) follows from part (c) and (E2.1.1).

If $V$ and $W$ are subspaces of a vector space $A$, let $V / W$ denote $V /(V \cap W)$.

DEFINITION 4.2.

(a) A subspace $V$ of a Lie algebra $L$ is said to have small centralizer if $($ ker $a d(x)) \cap$ $V$ has dimension 1 for all $x \in V \backslash\{0\}$.

(b) Let $Z$ be a Lie ideal of $L$. A subspace $V \subset L$ is said to have small centralizer modulo $Z$ if the quotient space $V / Z$ in $L / Z$ has small centralizer.

Proposition 4.3. Let $L$ be a coassociative Lie algebra and $Z$ be a Lie ideal of $L$. Suppose that $L^{\prime}$ and $L^{\prime \prime}$ are two subspaces of $L$ such that 
(a) $\left[L^{\prime}, Z\right]=\left[L^{\prime \prime}, Z\right]=0$,

(b) $L^{\prime}$ and $L^{\prime \prime}$ have small centralizers modulo $Z$ and

(c) $\delta(L) \subset L^{\prime \prime} \otimes L^{\prime}+(Z \otimes L+L \otimes Z)$.

Then $\operatorname{dim} \delta(L) /(Z \otimes L+L \otimes Z) \leq 1$.

REMARK 4.4. Since $Z$ is a Lie ideal of $L$, we have that $L / Z$ is a quotient Lie algebra, but $L / Z$ may not be a quotient of the coassociative Lie algebra $L$.

Proof of Proposition 4.3 Let $W=(Z \otimes L+L \otimes Z) \cap \delta(L)$. Without loss of generality we may assume that $\delta(L) \neq W$. Let $a$ and $b$ be any two elements in $L$. The assertion is equivalent to

Claim: $\delta(a)$ and $\delta(b)$ are linearly dependent in $\delta(L) / W$.

Proof of the Claim: If $\delta(a)$ or $\delta(b)$ is in $W$, the claim is obvious, so we assume during the proof that $\delta(a)$ and $\delta(b)$ are not in $W$.

In the rest of the proof, we will pick a $k$-linear basis $\left\{z_{j}\right\}_{j \geq 1}$ of $Z$, extend it to a basis $\left\{x_{i}\right\}_{i \geq 1} \cup\left\{z_{j}\right\}_{j \geq 1}$ of $L^{\prime \prime}+Z$ where $x_{i} \in L^{\prime \prime} \backslash Z$, then extend it to a basis $\left\{x_{i}\right\}_{i \geq 1} \cup$ $\left\{z_{j}\right\}_{j \geq 1} \cup\left\{l_{s}\right\}_{s \geq 1}$ of the whole space $L$ where $l_{s} \in L \backslash\left(L^{\prime \prime}+Z\right)$. For simplicity, we use integers to index the basis elements. Since $\delta(L) \subset L^{\prime \prime} \otimes L^{\prime}+(Z \otimes L+L \otimes Z)$, for any $a \in L$,

$$
\delta(a)=\sum_{i \geq 1} x_{i} \otimes a_{i}+\sum_{j \geq 1} z_{j} \otimes a_{j}^{\prime}+\sum_{s \geq 1} l_{s} \otimes a_{s}^{\prime \prime},
$$

where $a_{i} \in L^{\prime}+Z, a_{j}^{\prime} \in L$ and $a_{s}^{\prime \prime} \in Z$.

Case 1: Suppose that $\delta(a), \delta(b) \in u \otimes L^{\prime}+(Z \otimes L+L \otimes Z)$ for some $u \in L^{\prime \prime} \backslash Z$. In this case we can choose $x_{1}=u$. By the choice of the $k$-linear basis, we can write

$$
\delta(a)=u \otimes a_{1}+\sum_{i \geq 2} x_{i} \otimes a_{i}+\sum_{j \geq 1} z_{j} \otimes a_{j}^{\prime}+\sum_{s \geq 1} l_{s} \otimes a_{s}^{\prime \prime},
$$

where $a_{1} \in L^{\prime}+Z, a_{i} \in Z$ for all $i \geq 2, a_{j}^{\prime} \in L$ and $a_{s}^{\prime \prime} \in Z$ for all $j, s$. Similarly,

$$
\delta(b)=u \otimes b_{1}+\sum_{i \geq 2} x_{i} \otimes b_{i}+\sum_{j \geq 1} z_{j} \otimes b_{j}^{\prime}+\sum_{k \geq 1} l_{s} \otimes b_{s}^{\prime \prime},
$$

where $b_{1} \in L^{\prime}+Z, b_{i} \in Z$ for all $i \geq 2$, and $b_{j}^{\prime} \in L$ and $b_{s}^{\prime \prime} \in Z$ for all $j, s$. By Lemma 4.1(a), $\left[a_{1}, b_{1}\right]=0$. Since $L^{\prime}$ has small centralizer modulo $Z, b_{1} \in k a_{1}+Z$. Thus, $\delta(a)$ and $\delta(b)$ are linearly dependent in $\delta(L) / W$.

Case 2: Suppose that $\delta(a), \delta(b) \in L^{\prime \prime} \otimes u+(Z \otimes L+L \otimes Z)$ for some $u \in L^{\prime} \backslash Z$. Case 2 is equivalent to Case 1 by symmetry. Hence, the claim follows by Case 1 .

Case 3: Suppose that $\delta(a) \in u \otimes L^{\prime}+(Z \otimes L+L \otimes Z)$ for some $u \in L^{\prime \prime} \backslash Z$. In this case, we can choose $x_{1}=u$, and $\delta(a)$ can be written as in Case 1. In particular, $\delta(a) \in L^{\prime \prime} \otimes a_{1}+(Z \otimes L+L \otimes Z)$. Write

$$
\delta(b)=u \otimes b_{1}+\sum_{i \geq 2} x_{i} \otimes b_{i}+\sum_{j \geq 1} z_{j} \otimes b_{j}^{\prime}+\sum_{s \geq 1} l_{s} \otimes b_{s}^{\prime \prime},
$$

where $b_{i} \in L^{\prime}+Z$ for all $i \geq 1$, and $b_{j}^{\prime} \in L, b_{s}^{\prime \prime} \in Z$ for all $j, s$. By Lemma 4.1(a), $\left[a_{1}, b_{1}\right]=0$. Since $L^{\prime}$ has small centralizer modulo $Z, b_{1}=\lambda a_{1}+z$ for some $\lambda \in k$ and $z \in Z$. Replacing $b_{1}$ by $b_{1}-\lambda a_{1}$, we may assume that $b_{1} \in Z$. By Lemma 4.1(b), 
for every $i \geq 2,\left[a_{1}, b_{i}\right]=-\left[a_{i}, b_{1}\right] \in Z$ since $b_{1}$ is in $Z$. Since $L^{\prime \prime}$ has small centralizer modulo $Z, b_{i}=\lambda_{i} a_{1}+z_{i}$, for $\lambda_{i} \in k$ and $z_{i} \in Z$ for all $i \geq 2$. Thus,

$$
\delta(b)=u \otimes b_{1}+\sum_{i \geq 2} x_{i} \otimes\left(\lambda_{i} a_{1}+z_{i}\right)+\sum_{j \geq 1} z_{j} \otimes b_{j}^{\prime}+\sum_{s \geq 1} l_{s} \otimes b_{s}^{\prime \prime},
$$

which is in $L^{\prime \prime} \otimes a_{1}+(Z \otimes L+L \otimes Z)$. Therefore, both $\delta(a)$ and $\delta(b)$ are in $L^{\prime \prime} \otimes a_{1}+$ $(Z \otimes L+L \otimes Z)$. The claim follows from Case 2 .

Case 4: Suppose that either $\delta(a)$ or $\delta(b)$ is in $L^{\prime \prime} \otimes u+(Z \otimes L+L \otimes Z)$ for some $u \in L^{\prime} \backslash Z$. The claim follows by symmetry and Case 3 .

Case 5 (the general case): By the choice of $k$-linear basis, we can write

$$
\begin{aligned}
& \delta(a)=\sum_{i \geq 1} x_{i} \otimes a_{i}+\sum_{j \geq 1} z_{j} \otimes a_{j}^{\prime}+\sum_{s \geq 1} l_{s} \otimes a_{s}^{\prime \prime}, \\
& \delta(b)=\sum_{i \geq 1} x_{i} \otimes b_{i}+\sum_{j \geq 1} z_{j} \otimes b_{j}^{\prime}+\sum_{s \geq 1} l_{s} \otimes b_{s}^{\prime \prime},
\end{aligned}
$$

where $a_{i}, b_{i} \in L^{\prime}+Z, a_{j}^{\prime}, b_{j}^{\prime} \in L$ and $a_{s}^{\prime \prime}, b_{s}^{\prime \prime} \in Z$ for all $i, j, s$. Without loss of generality, we may assume that $a_{1} \in L^{\prime} \backslash Z$. By Lemma 4.1(a), $\left[a_{1}, b_{1}\right]=0$. Since $L^{\prime}$ has small centralizer modulo $Z, b_{1}=\lambda a_{1}+z$ for some $\lambda \in k$ and where $z \in Z$. Replacing $b_{1}$ by $b_{1}-\lambda a_{1}$, we may assume that $b_{1} \in Z$. By Lemma 4.1(b), for every $i \geq 2,\left[a_{1}, b_{i}\right]=$ $-\left[a_{i}, b_{1}\right] \in Z$ since $b_{1}$ are in the Lie ideal $Z$. Since $L^{\prime}$ has small centralizer modulo $Z$, $b_{i}=\lambda_{i} a_{1}+z_{i}$ for $\lambda_{i} \in k$ and $z_{i} \in Z$ for all $i \geq 2$. Together with the fact $b_{1} \in Z$, we have that $\delta(b) \in L^{\prime \prime} \otimes a_{1}+(Z \otimes L+L \otimes Z)$. The claim now follows from Case 4 .

For a subset $S \subset L$, the centralizer of $S$ in $L$ is defined to be

$$
C_{S}(L)=\{y \in L \mid[x, y]=0, \forall x \in S\} .
$$

LEMMA 4.5. If there is an element $a \in L$ such that $\delta(a)=x \otimes y \neq 0$, then $\delta(L) \subset$ $C_{\{x\}}(L) \otimes C_{\{y\}}(L)$.

Proof. By symmetry, it suffices to show that $\delta(L) \subset L \otimes C_{\{y\}}(L)$. Pick a basis $\left\{x_{i}\right\}$ of $L$ such that $x_{1}=x$. Then $\delta(a)=x_{1} \otimes y$. For any $b \in L$, write $\delta(b)=\sum_{i} x_{i} \otimes b_{i}$. By Lemma $4.1(\mathrm{a}),\left[y, b_{1}\right]=0$. For any $i \geq 2$, by Lemma $4.1(\mathrm{~b}),\left[y, b_{i}\right]=-\left[0, b_{1}\right]=0$. The assertion follows.

Proposition 4.6. Let $C$ be the co-radical of a coassociative Lie algebra L. Then $[C, C]=0$. As a consequence, cosemi-simple coalgebras are rigid.

Proof. Since $k$ is algebraically closed, $C=\bigoplus_{i} M_{n_{i}}(k)$ for a set of positive integers $\left\{n_{i}\right\}_{i \in I}$. Let $x, y \in C$; we need to show $[x, y]=0$. By linearity, we may assume that $x$ and $y$ are some basis elements in $C$. We need to consider two cases.

Case 1: We have that $x$ and $y$ are in the same matrix subcoalgebra, say $M_{n}(k)$. If $n=1, x=y$. The assertion is trivial. Now assume that $n>1$. Then we may assume that $x=x_{i j}$ and $y=x_{k l}$ for some $i, j, k, l$. Consider $\delta\left(x_{1 j}\right)=\sum_{s} x_{1 s} \otimes x_{s j}$ and $\delta\left(x_{2 l}\right)=$ $\sum_{t} x_{2 t} \otimes x_{t l}$. By Lemma 4.1(b), $\left[x_{s j}, x_{t l}\right]=0$ for all $s, t$. The assertion follows.

Case 2: We have that $x$ and $y$ are in different matrix subcoalgebras. Then we may assume that $x=x_{i j} \in M_{n_{1}}(k)$ and $y=y_{k l} \in M_{n_{2}}(k)$. Consider $\delta\left(x_{1 j}\right)=\sum x_{1 s} \otimes$ $x_{s j}$ and $\delta\left(y_{1 l}\right)=\sum y_{1 t} \otimes y_{t l}$. By Lemma 4.1(b), $\left[x_{s j}, y_{t l}\right]=0$ for all $s, t$. The assertion follows. 
The following lemma is also true. The proof is omitted since it is straightforward and somewhat similar to the proof of Proposition 4.6.

LEMMA 4.7. If $C_{1}$ and $C_{2}$ are subcoalgebras of a coassociative Lie algebra such that $C_{1} \cap C_{2}=\{0\}$, then $\left[\delta\left(C_{1}\right), \delta\left(C_{2}\right)\right]=0$.

LEMMA 4.8. Let L be a coalgebra. Then we have the following statements.

(a) For every $x \in L$, write $\delta(x)=\sum_{i=1}^{n} w_{i} \otimes v_{i}$ for a minimal $n$. Then $\sum_{i} k \delta\left(v_{i}\right) \subset$ $L \otimes V_{x}$ and $\sum_{i} k \delta\left(w_{i}\right) \subset W_{x} \otimes L$ for some subspaces $V_{x} \subset \sum_{i} k v_{i}$ and $W_{x} \subset$ $\sum_{i} k w_{i}$ of dimension no more than $\operatorname{dim} \delta(L)$.

(b) If $\delta(L)$ is 1-dimensional and $L$ is not 2-conilpotent, then $\delta(L)$ has a basis element of the form $T \otimes T$ for some $0 \neq T \in L$.

Proof. (a) Let $\left\{y_{t}\right\}_{t=1}^{m}$ be a basis of $\sum_{s} k \delta\left(v_{s}\right)$ for some $m \leq \operatorname{dim} \delta(L)$. Then there are elements $a_{1}, \ldots, a_{m} \in L$ such that

$$
\sum_{i} \delta\left(w_{i}\right) \otimes v_{i}=\sum_{i} w_{i} \otimes \delta\left(v_{i}\right)=\sum_{t=1}^{m} a_{t} \otimes y_{t} \in\left(\sum_{t=1}^{m} k a_{t}\right) \otimes L \otimes L .
$$

This implies that $\delta\left(w_{i}\right) \in\left(\sum_{t} k a_{t}\right) \otimes L$ for each $i$. Since $\left\{y_{t}\right\}_{t=1}^{m}$ is a basis of $\sum_{s} k \delta\left(v_{s}\right)$, the equation

$$
\sum_{i} w_{i} \otimes \delta\left(v_{i}\right)=\sum_{t=1}^{m} a_{t} \otimes y_{t}
$$

implies that $\sum_{t} k a_{t} \subset \sum_{i} k w_{i}$. Therefore, the second assertion follows by taking $W_{x}=$ $\sum_{t} k a_{t}$. The first assertion is similar.

(b) Pick $0 \neq \Omega \in \delta(L)$, and let $\left\{x_{i}\right\}$ be a finite set of linearly independent elements in $L$ such that $\Omega=\sum_{i, j} a_{i j} x_{i} \otimes x_{j}$. Since $\delta(L)$ is 1-dimensional, $\delta\left(x_{i}\right)=b_{i} \Omega$ for some $b_{i} \in k$. Pick $x \in L$ such that $\delta(x)=\Omega$ and $(\delta \otimes 1) \delta(x) \neq 0$ (since $L$ is not 2-conilpotent). Then,

$$
(\delta \otimes 1) \delta(x)=\sum_{i, j} a_{i, j} b_{i} \Omega \otimes x_{j}=\Omega \otimes\left(\sum_{i, j} a_{i j} b_{i} x_{j}\right)=\Omega \otimes T,
$$

where $T:=\sum_{i, j} a_{i j} b_{i} x_{j} \in L$, and

$$
(1 \otimes \delta) \delta(x)=\sum_{i, j} a_{i j} x_{i} \otimes b_{j} \Omega=\left(\sum_{i, j} a_{i j} b_{j} x_{i}\right) \otimes \Omega=S \otimes \Omega,
$$

where $S:=\sum_{i, j} a_{i j} b_{j} x_{i}$. By coassociativity, $\Omega \otimes T=S \otimes \Omega$. This implies that $\Omega=$ $c^{\prime} T \otimes T$ and $S=c^{\prime \prime} T$ for some $c^{\prime}, c^{\prime \prime} \in k^{\times}$. Since $k$ is algebraically closed, we can choose $c^{\prime}=1$ by a scalar change of $T$.

Now we prove the main result of this section. Let

$$
\left\{e:=\left(\begin{array}{ll}
0 & 1 \\
0 & 0
\end{array}\right), \quad f:=\left(\begin{array}{ll}
0 & 0 \\
1 & 0
\end{array}\right), \quad h:=\left(\begin{array}{cc}
1 & 0 \\
0 & -1
\end{array}\right)\right\}
$$

be a standard $k$-basis of $s l_{2}$. 
THEOREM 4.9. The simple Lie algebra $\mathrm{sl}_{2}$ is rigid.

Proof. By using the standard basis of $s l_{2}$, it is straightforward to check that $s l_{2}$ has small centralizers (details are omitted). Let $L=\left(s l_{2}, \delta\right)$ be a coassociative Lie algebra. We need to show that $\delta=0$. By Proposition 4.3 for $L^{\prime}=L^{\prime \prime}=L=s l_{2}$ and $Z=0$, $\operatorname{dim} \delta(L) \leq 1$. To avoid the triviality, we assume that $\operatorname{dim} \delta(L)=1$ and let $\Omega \in \delta(L)$ be a non-zero element.

If $L$ is not 2-conilpotent, then Lemma 4.8 says that $\Omega=T \otimes T$, where $T=t_{1} e+$ $t_{2} f+t_{3} h$ for some $t_{1}, t_{2}, t_{3} \in k$. Since $\Omega \neq 0$, not all $t_{i}$ are zero. Suppose $\delta(e)=a \Omega$, $\delta(f)=b \Omega$ and $\delta(h)=c \Omega$ for some $a, b, c \in k$. By (E2.1.1), we have

$$
\begin{aligned}
2 b \Omega= & \delta(2 f)=\delta([f, h]) \\
= & {[f \otimes 1+1 \otimes f, c \Omega]+[b \Omega, h \otimes 1+1 \otimes h] } \\
= & \left\{-2 b t_{1} e+\left(2 c t_{3}+2 b t_{2}\right) f-c t_{1} h\right\} \otimes T \\
& +T \otimes\left\{-2 b t_{1} e+\left(2 c t_{3}+2 b t_{2}\right) f-c t_{1} h\right\} .
\end{aligned}
$$

Hence, $b\left(t_{1}, t_{2}, t_{3}\right)=\left(-2 b t_{1}, 2 b t_{2}+2 c t_{3},-c t_{1}\right)$, or

$$
M\left(\begin{array}{l}
t_{1} \\
t_{2} \\
t_{3}
\end{array}\right)=0 \text { where } M=\left(\begin{array}{ccc}
b & 0 & 0 \\
0 & b & 2 c \\
c & 0 & b
\end{array}\right)
$$

Since not all $t_{1}, t_{2}, t_{3}$ are zero, the determinant of matrix $M$, which is $b^{3}$, is zero. Hence, $b=0$. Since $e$ and $f$ play a very similar role, by symmetry, $a=0$. By (E2.1.1) and the fact $\delta(e)=\delta(f)=0$, we have that $\delta(h)=\delta([e, f])=0$. Thus, $c=0$, whence $\delta=0$, yielding a contradiction. Therefore, $\delta$ is 2-conilpotent.

Since $\delta$ is 2-conilpotent, by Theorem 3.5, $U\left(s l_{2}, \delta\right)$ is a connected Hopf algebra of GKdim 3. By comparing with the list in the classification of connected Hopf algebras of GKdim 3 [10, Theorem 1.3], $U\left(s l_{2}, \delta\right)$ must be isomorphic to $U\left(s l_{2}\right)$. Since $U\left(s l_{2}\right)$ is cocommutative, $\left(s l_{2}, \delta\right)$ must be cocommutative. Hence,

$$
\begin{aligned}
\Omega= & a_{11} e \otimes e+a_{12}(e \otimes f+f \otimes e)+a_{13}(e \otimes h+h \otimes e) \\
& +a_{22} f \otimes f+a_{23}(f \otimes h+h \otimes f)+a_{33} h \otimes h \neq 0 .
\end{aligned}
$$

Since $\delta(L)$ is 1-dimensional, the kernel $L_{0}=\operatorname{ker}(\delta)$ is a 2-dimensional Lie subalgebra of $s l_{2}$. By an elementary computation, it is easy to verify that any 2-dimensional Lie subalgebra of $s l_{2}$ is either (i) $k f+k h$ or (ii) $k e+k h$ or (iii) $k(e+a h)+k(-4 a f+h)$ for some $a \in k^{\times}$. In the first case, $(\delta \otimes 1) \Omega=a_{11} \delta(e) \otimes e+a_{12} \delta(e) \otimes f+a_{13} \delta(e) \otimes h$ and without loss of generality, we can assume that $\delta(e)=\Omega$. Since $\left(s l_{2}, \delta\right)$ is 2-conilpotent, $(\delta \otimes 1)(\Omega)=0$, which implies that $a_{11}=a_{12}=a_{13}=0$. It is easy to see that

$$
\begin{aligned}
-\Phi(e, h) & =-\delta(-2 e)+[\delta(e), h \otimes 1+1 \otimes h] \\
& =2 \Omega+4 a_{22} f \otimes f+2 a_{23}(f \otimes h+h \otimes f) .
\end{aligned}
$$

Equations $\Phi(e, h)=[\delta(e), \delta(h)]=[\delta(e), 0]=0$ imply that $a_{22}=a_{23}=a_{33}=0$. Hence, $\Omega=0$, a contradiction. The assertion follows. The second case is similar. 
The final case is when $e+a h, h-4 a f \in \operatorname{ker}(\delta)$ for some $a \in k^{\times}$. Let

$$
\begin{aligned}
e^{\prime} & =\left(\begin{array}{cc}
1 & 0 \\
-2 a & 1
\end{array}\right)\left(\begin{array}{cc}
0 & 1 \\
0 & 0
\end{array}\right)\left(\begin{array}{cc}
1 & 0 \\
-2 a & 1
\end{array}\right)^{-1}=\left(\begin{array}{cc}
2 a & 1 \\
-4 a^{2} & -2 a
\end{array}\right) \\
h^{\prime} & =\left(\begin{array}{cc}
1 & 0 \\
-2 a & 1
\end{array}\right)\left(\begin{array}{cc}
1 & 0 \\
0 & -1
\end{array}\right)\left(\begin{array}{cc}
1 & 0 \\
-2 a & 1
\end{array}\right)^{-1}=\left(\begin{array}{cc}
1 & 0 \\
-4 a & -1
\end{array}\right) \\
f^{\prime} & =\left(\begin{array}{cc}
1 & 0 \\
-2 a & 1
\end{array}\right)\left(\begin{array}{cc}
0 & 0 \\
1 & 0
\end{array}\right)\left(\begin{array}{cc}
1 & 0 \\
-2 a & 1
\end{array}\right)^{-1}=\left(\begin{array}{cc}
0 & 0 \\
1 & 0
\end{array}\right)=f .
\end{aligned}
$$

Then $\left\{e^{\prime}, f^{\prime}, h^{\prime}\right\}$ is a new standard basis of $s l_{2}$. It is clear that $e^{\prime}=e+2 a h-4 a^{2} f \in$ $\operatorname{ker}(\delta)$ and $h^{\prime}=h-4 a f \in \operatorname{ker}(\delta)$. Thus, it is equivalent to the second case. Combining all these cases, the assertion follows.

Similar to Theorem 4.9, we show the following.

THEOREM 4.10. Write $g l_{2}=s l_{2} \oplus k z$, where $k z$ is the centre of $g l_{2}$. If $\left(g l_{2}, \delta\right)$ is a coassociative Lie algebra, then $\left.\delta\right|_{s l_{2}}=0$ and $\delta(z)=a z \otimes z$ for some scalar $a \in k$.

Sketch of proof. Some tedious computations are omitted in the following proof.

First of all, the $\delta$ given in the theorem gives rise to a coassociative Lie algebra structure on $g l_{2}$. Now we assume that $\left(g l_{2}, \delta\right)$ is a coassociative Lie algebra.

Applying Proposition 4.3 to $\left(L, L^{\prime}, L^{\prime \prime}, Z\right)=\left(g l_{2}, s l_{2}, s l_{2}, k z\right)$, we obtain that

$$
\operatorname{dim}\left(\delta\left(g l_{2}\right) /\left(z \otimes g l_{2}+g l_{2} \otimes z\right)\right) \leq 1 .
$$

Therefore, there is an element $\Omega \in s l_{2} \otimes s l_{2}$ such that, for every $x \in g l_{2}$,

$$
\delta(x)=\sigma(x) \otimes z+z \otimes \tau(x)+\lambda(x) \Omega
$$

for some $\sigma(x) \in s l_{2}, \tau(x) \in g l_{2}$ and $\lambda(x) \in k$. Both $\sigma(x)$ and $\tau(x)$ are uniquely determined by (E4.10.1). If $\Omega \neq 0$, then $\lambda(x)$ is also uniquely determined by (E4.10.1). Setting $x=z$ in (E4.10.1), we have

$$
\delta(z)=\sigma(z) \otimes z+z \otimes \tau(z)+\lambda(z) \Omega .
$$

Equation (E2.2.2) for $(a, b)=(z, x)$ implies that

$$
\begin{aligned}
0= & {[\sigma(z), x] \otimes z+z \otimes[\tau(z), x]+[\lambda(z) \Omega, x \otimes 1+1 \otimes x] } \\
& +[\sigma(z), \sigma(x)] \otimes z^{2}+z^{2} \otimes[\tau(z), \tau(x)] \\
& +[\lambda(z) \Omega, \sigma(x) \otimes z]+[\lambda(z) \Omega, z \otimes \tau(x)] \\
& +[\sigma(z) \otimes z, \lambda(x) \Omega]+[z \otimes \tau(z), \lambda(x) \Omega] .
\end{aligned}
$$

Since the terms in the above equation live in different $k$-subspaces of $U\left(g l_{2}\right) \otimes U\left(g l_{2}\right)$, we have $[\sigma(z), x]=0=[\tau(z), x]$ for all $x \in s l_{2}$. Thus, $\sigma(z)=0$ and $\tau(z) \in k z$. In this case, (E2.2.2) becomes, for every $x \in g l_{2}$,

$$
0=\lambda(z)([\Omega, x \otimes 1+1 \otimes x]+[\Omega, \sigma(x) \otimes z]+[\Omega, z \otimes \tau(x)]) .
$$

First we claim that $\lambda(z) \Omega=0$. If not, we may assume that $\Omega \neq 0$ and $\lambda(z)=1$. Using the fact that terms live in different $k$-subspaces of $U\left(g l_{2}\right) \otimes U\left(g l_{2}\right),(\mathrm{E} 4.10 .3)$ 
implies that

$$
[\Omega, x \otimes 1+1 \otimes x]=[\Omega, \sigma(x) \otimes z]=[\Omega, z \otimes \tau(x)]=0
$$

for all $x \in g l_{2}$. A computation shows that the first equation implies that $\Omega=c(h \otimes$ $h+2(e \otimes f+f \otimes e))$ for some $0 \neq c \in k$. The second and third equations imply that $\sigma(x)=0$ and $\tau(x) \in k z$. Going back to (E4.10.1), we have, for every $x \in g l_{2}$,

$$
\delta(x)=\phi(x) z \otimes z+\lambda(x) \Omega
$$

for some $\phi(x) \in k$. For any $x, y \in s l_{2},(\mathrm{E} 2.2 .2)$ says that

$$
\delta([x, y])=[x \otimes 1+1 \otimes x, \lambda(y) \Omega]+[\lambda(x) \Omega, y \otimes 1+1 \otimes y]=0 .
$$

Since $s l_{2}=\left[s l_{2}, s l_{2}\right],\left.\delta\right|_{s l_{2}}=0$. The coassociativity on $z$ shows that $\Omega \otimes z=0$, a contradiction. Therefore, we proved our claim.

For the rest of the proof, we have $\lambda(z) \Omega=0$ and $\delta(z)=\sigma(z) \otimes z+z \otimes \tau(z)$. Then $k z$ is an ideal of the coassociative Lie algebra $\left(g l_{2}, \delta\right)$ and $\left(g l_{2} / k z, \bar{\delta}\right) \cong\left(s l_{2}, \bar{\delta}\right)$ is a quotient coassociative Lie algebra where $\bar{\delta}$ is the induced coproduct. By Theorem 4.9, $\bar{\delta}=0$. This means that $\Omega=0$. For each $x \in g l_{2}$, write

$$
\delta(x)=\sigma_{1}(x) \otimes z+z \otimes \sigma_{2}(x)+v(x) z \otimes z
$$

for some $\sigma_{1}(x), \sigma(x) \in s l_{2}$ and some $v(x) \in k$. By (E2.2.2), we have

$$
\begin{aligned}
\delta([x, y])= & {\left[\sigma_{1}(x), y\right] \otimes z+z \otimes\left[\sigma_{2}(x), y\right]+\left[x, \sigma_{1}(y)\right] \otimes z+z \otimes\left[x, \sigma_{2}(y)\right] } \\
& +\left[\sigma_{1}(x), \sigma_{1}(y)\right] \otimes z^{2}+z^{2} \otimes\left[\sigma_{2}(x), \sigma_{2}(y)\right]
\end{aligned}
$$

for all $x, y \in g l_{2}$. Setting $y=z$, we have $\left[x, \sigma_{i}(z)\right]=0$ for all $x \in s l_{2}$. This implies that $\sigma_{i}(z)=0$ for $i=1,2$. Setting $x, y \in s l_{2}$, we have that

$$
\left[\sigma_{1}(x), \sigma_{1}(y)\right]=0=\left[\sigma_{2}(x), \sigma_{2}(y)\right]
$$

and that $\delta\left(s l_{2}\right) \subset s l_{2} \otimes z+z \otimes s l_{2}$. Since $s l_{2}$ has small centralizers, $\operatorname{dim} \sigma_{1}\left(s l_{2}\right) \leq 1$ and $\operatorname{dim} \sigma_{2}\left(s l_{2}\right) \leq 1$. Combining above facts, there exist $w_{1}, w_{2} \in s l_{2} \backslash\{0\}, c \in k$ and linear maps $\phi_{1}, \phi_{2}: s l_{2} \rightarrow k$, such that

$$
\begin{aligned}
& \delta(z)=c z \otimes z, \\
& \delta(x)=\phi_{1}(x) w_{1} \otimes z+\phi_{2}(x) z \otimes w_{2}
\end{aligned}
$$

for all $x \in s l_{2}$. Let $\{e, f, h\}$ be the standard basis of $s l_{2}$, and write $w_{1}=a e+b f+c h \neq 0$ for some $a, b, c \in k$. A calculation using explicit Lie product of the elements $e, f$ and $h$ shows that (E2.2.2) implies that $\phi_{1}=0$. By symmetry, $\phi_{2}=0$. Thus, the assertion follows.

5. Examples. We present several families of coassociative Lie algebras in this section. One-dimensional families are listed in Example 2.9. Here is the 2-dimensional case.

EXAMPLE 5.1. If $\operatorname{dim} L=2$, then there are two Lie algebra structures on $L$ up to isomorphism. Namely, $L$ is either abelian or non-abelian. 
If $L$ is abelian, then the classification of coassociative Lie algebra structures on $L$ is equivalent to the classification of coalgebra structures on $L$. It is easy to show that $\delta$-structure in $L$ is isomorphic to one of the following:

(5.1.1) $\delta=0$;

(5.1.2) $(L, \delta)$ is cosemi-simple;

(5.1.3) $L=k x_{1} \oplus k x_{2}$ and $\delta\left(x_{1}\right)=x_{1} \otimes x_{1}, \delta\left(x_{2}\right)=0$;

(5.1.4) $L=k x_{1} \oplus k x_{2}$ and $\delta\left(x_{1}\right)=x_{1} \otimes x_{1}, \delta\left(x_{2}\right)=x_{1} \otimes x_{2}+x_{2} \otimes x_{1}$;

(5.1.5) $L=k x_{1} \oplus k x_{2}$ and $\delta\left(x_{1}\right)=0, \delta\left(x_{2}\right)=x_{1} \otimes x_{1}$.

If $L$ is non-abelian, then $L$ has a basis $\left\{x_{1}, x_{2}\right\}$ such that $\left[x_{1}, x_{2}\right]=x_{2}$. We have the following two cases.

Case 1: $\delta\left(x_{2}\right)=0$. We are only interested in non-zero $\delta$-structures. Write $\delta\left(x_{1}\right)=$ $\sum_{i, j} a_{i j} x_{i} \otimes x_{j} \neq 0$. In this case

$$
\Phi\left(x_{1}, x_{2}\right)=-\left[\delta\left(x_{1}\right), x_{2} \otimes 1+1 \otimes x_{2}\right]=-\left(a_{11}\left(x_{2} \otimes x_{1}+x_{1} \otimes x_{2}\right)+\left(a_{12}+a_{21}\right) x_{2} \otimes x_{2}\right) .
$$

By (E2.3.2), $\Phi\left(x_{1}, x_{2}\right)=\left[\delta\left(x_{1}\right), \delta\left(x_{2}\right)\right]=0$. Hence, $a_{11}=0$ and $a_{12}+a_{21}=0$. Let $a=a_{12}$ and $b=a_{22}$. We have that $\delta\left(x_{1}\right)=a x_{1} \otimes x_{2}-a x_{2} \otimes x_{1}+b x_{2} \otimes x_{2}$. Now coassociativity of $\delta$ shows that $a=0$. Thus, $\delta\left(x_{2}\right)=0$ and $\delta\left(x_{1}\right)=b x_{2} \otimes x_{2}$. Up to a base change, we may assume $b=1$. Hence, this is Example 2.4.

Case 2: $\delta\left(x_{2}\right) \neq 0$. Since $L$ is 2-dimensional and non-nilpotent, it has small centralizers. By Lemma 4.3, $\delta(L)$ is 1-dimensional. Since $\delta\left(x_{2}\right) \neq 0$, by replacing $x_{1}$ by $x_{1}-a x_{2}$ for some suitable $a \in k$, we have $\delta\left(x_{1}\right)=0$. Write $\delta\left(x_{2}\right)=\sum_{i, j} b_{i j} x_{i} \otimes x_{j} \neq 0$. In this case,

$$
\begin{aligned}
0=\left[\delta\left(x_{1}\right), \delta\left(x_{2}\right)\right]= & \Phi\left(x_{1}, x_{2}\right)=\delta\left(x_{2}\right)-\left[x_{1} \otimes 1+1 \otimes x_{1}, \delta\left(x_{2}\right)\right] \\
= & b_{11} x_{1} \otimes x_{1}+b_{12} x_{1} \otimes x_{2}+b_{21} x_{2} \otimes x_{1}+b_{22} x_{2} \otimes x_{2} \\
& -\left(b_{12} x_{1} \otimes x_{2}+b_{21} x_{2} \otimes x_{1}+2 b_{22} x_{2} \otimes x_{2}\right) \\
= & b_{11} x_{1} \otimes x_{1}-b_{22} x_{2} \otimes x_{2} .
\end{aligned}
$$

Hence, $b_{11}=b_{22}=0$ and $\delta\left(x_{2}\right)=b_{12} x_{1} \otimes x_{2}+b_{21} x_{2} \otimes x_{1}$. The coassociativity of $\delta$ implies that $b_{12}=b_{21}=0$. Therefore, $\delta=0$ in this case, yielding a contradiction.

Combining these two cases, the only non-zero $\delta$-structure on the 2-dimensional non-abelian Lie algebra is the one in Example 2.4 up to isomorphisms.

One nice fact in 2-dimensional case is that $\delta$ is always cocommutative. If $\delta$ is conilpotent, then $L$ is quasi-equivalent to a Lie algebra by Corollary 3.6.

Next, we consider some higher dimensional examples.

EXAMPLE 5.2. Let $\mathfrak{g}$ be a 3 -dimensional Lie algebra with a $k$-linear basis $\{x, y, z\}$ such that its Lie structure is determined by

$$
[x, y]=y, \quad[z, y]=0, \quad[z, x]=-z+\lambda y,
$$

for any $\lambda \in k$. Let $L=(\mathfrak{g}, \delta)$, where the coproduct $\delta$ is determined by

$$
\delta(x)=\delta(y)=0, \quad \delta(z)=x \otimes y-y \otimes x .
$$

It is routine to check that $L$ is a coassociative Lie algebra (using Definition 2.1). It is obvious that $\delta$ is conilpotent and anti-cocommutative. Let $H$ be the enveloping algebra $U(L)$. It follows from the antipode axiom that $S(x)=-x, S(y)=-y$, and 
$S(z)=-z+y$. Hence, $S^{2}(z)=z-2 y$ and $H$ is not involutory. By Theorem $3.7, \mathfrak{g}$ is not unimodular, which can also be verified directly.

Let $\mathfrak{h}_{2 n+1}$ be the $(2 n+1)$-dimensional Heisenberg Lie algebra with a standard basis $\left\{x_{1}, \cdots, x_{n}, y_{1}, \cdots, y_{n}, z\right\}$. Here $\left[x_{i}, y_{i}\right]=z$ for all $i$, and all other brackets are zero. Let $A=\left(a_{i j}\right), B=\left(b_{i j}\right), C=\left(c_{i j}\right)$ and $D=\left(d_{i j}\right)$ denote $n \times n$-matrices over $k$, and let $E=\left(e_{i}\right)$ be an $n$-column vector over $k$.

EXAMPLE 5.3. Each of the following $\delta$ defines a coassociative coalgebra structure on $\mathfrak{h}_{2 n+1}$ such that $\left(\mathfrak{h}_{2 n+1}, \delta\right)$ is a coassociative Lie algebra.

(a) For every $i, \delta\left(x_{i}\right)=\delta(z)=0$, and

$$
\delta\left(y_{i}\right)=\sum_{j}\left(a_{i j} x_{j}+b_{i j} y_{j}\right) \otimes z+\sum_{j} z \otimes\left(c_{i j} x_{j}-b_{i j} y_{j}\right)+e_{i} z \otimes z,
$$

where the coefficient matrices $A=\left(a_{i j}\right), B=\left(b_{i j}\right), C=\left(c_{i j}\right)$ and $E=\left(e_{i}\right)$ satisfy

(i) $B A=B^{2}=B C=0$;

(ii) $B E=0$;

(iii) $A+A^{\tau}+C+C^{\tau}=0$;

(iv) $A B^{\tau}=B A^{\tau}$ and

(v) $C B^{\tau}=B C^{\tau}$.

(b) For every $i, \delta\left(x_{i}\right)=e_{i} z \otimes z, \delta(z)=0$ and $\delta\left(y_{i}\right)=\sum b_{i j}\left(x_{j} \otimes z+z \otimes x_{j}\right)$, where the coefficient matrix $B=\left(b_{i j}\right)$ satisfies $B=B^{\tau}$.

(c) For every $i, \delta\left(x_{i}\right)=0, \delta(z)=z \otimes z$, and

$$
\delta\left(y_{i}\right)=\sum_{j}\left(a_{i j} x+b_{i j} y_{j}\right) \otimes z+\sum_{j} z \otimes\left(-a_{j i} x_{j}+\left(\delta_{i j}-b_{i j}\right) y_{j}\right),
$$

where the coefficient matrices $A=\left(a_{i j}\right)$ and $B=\left(b_{i j}\right)$ satisfy the conditions $B^{2}=B ; B A=A$ and $B A^{\tau}=0$.

Proof. (a) Easy computations show that

$(\delta \otimes 1) \delta\left(y_{i}\right)$

$$
\begin{aligned}
= & \sum_{j} b_{i j} \delta\left(y_{j}\right) \otimes z \\
= & \sum_{j, s}\left(b_{\ddot{i}} a_{j s} x_{s}+b_{i j} b_{j s} y_{s}\right) \otimes z \otimes z+\sum_{j, s} z \otimes\left(b_{i j} c_{j s} x_{s}-b_{i j} b_{j s} y_{s}\right) \otimes z \\
& +\sum_{j} b_{i j} e_{j} z \otimes z \otimes z, \quad \text { and }
\end{aligned}
$$

$(1 \otimes \delta) \delta\left(y_{i}\right)$

$$
\begin{aligned}
= & \sum_{j} z \otimes\left(-b_{i j}\right) \delta\left(y_{j}\right) \\
= & \sum_{j, s} z \otimes\left(-b_{i j} a_{j s} x_{s}-b_{i j} b_{j s} y_{s}\right) \otimes z+\sum_{j, s} z \otimes z \otimes\left(-b_{i j} c_{j s} x_{s}+b_{i j} b_{j s} y_{s}\right) \\
& -\sum_{j} b_{i j} e_{j} z \otimes z \otimes z .
\end{aligned}
$$


Coassociativity is equivalent to equations $B A=B^{2}=B C=0$ and $B E=0$. To check condition (E2.1.1) we note that (E2.1.1) is trivial when $(a, b)=(z, z),\left(x_{i}, z\right),\left(y_{i}, z\right)$ and $\left(x_{i}, x_{j}\right)$. It suffices to verify (E2.1.1) for $(a, b)=\left(x_{i}, y_{j}\right)$ and $(a, b)=\left(y_{i}, y_{j}\right)$ for all $1 \leq i, j \leq n$.

If $(a, b)=\left(x_{i}, y_{j}\right)$, we have that

$$
\begin{aligned}
& \text { LHS of }(\mathrm{E} 2.1 .1)=\delta\left(\left[x_{i}, y_{j}\right]\right)=\delta\left(\delta_{i j} z\right)=0, \text { and } \\
& \text { RHS of }(\mathrm{E} 2.1 .1)=\left[x_{i} \otimes 1+1 \otimes x_{i}, \delta\left(y_{j}\right)\right]=b_{j i} z \otimes z-b_{j i} z \otimes z=0 .
\end{aligned}
$$

Hence, $(\mathrm{E} 2.1 .1)$ holds for $(a, b)=\left(x_{i}, y_{j}\right)$.

If $(a, b)=\left(y_{i}, y_{j}\right)$, we have that

$$
\begin{aligned}
\text { LHS of }(\mathrm{E} 2.1 .1)= & \delta\left(\left[y_{i}, y_{j}\right]\right)=\delta(0)=0, \text { and } \\
\operatorname{RHS} \text { of }(\mathrm{E} 2.1 .1)= & {\left[y_{i} \otimes 1, \delta\left(y_{j}\right)\right]+\left[1 \otimes y_{i}, \delta\left(y_{j}\right)\right] } \\
& +\left[\delta\left(y_{i}\right), y_{j} \otimes 1\right]+\left[\delta\left(y_{i}\right), 1 \otimes y_{j}\right]+\left[\delta\left(y_{i}\right), \delta\left(y_{j}\right)\right] \\
= & a_{j i} z \otimes z+c_{j i} z \otimes z \\
& +a_{i j} z \otimes z+c_{i j} z \otimes z \\
& +\left(\sum_{s} a_{i s} b_{j s}-b_{i s} a_{j s}\right) z \otimes z^{2}+\left(\sum_{s}-c_{i s} b_{j s}+b_{i s} c_{j s}\right) z^{2} \otimes z .
\end{aligned}
$$

Hence, (E2.1.1) holds if and only if $A+A^{\tau}+C+C^{\tau}=0, A B^{\tau}=B A^{\tau}$ and $C B^{\tau}=$ $B C^{\tau}$. This completes the proof of (a).

The proofs of (b) and (c) are similar and therefore omitted.

We consider one last example. Let $U_{n}$ be the strictly upper triangular $n \times n$-matrix coalgebra, namely it is the coalgebra with basis $\left\{x_{i j}\right\}_{1 \leq i<j \leq n}$ such that

$$
\delta\left(x_{i j}\right)=\sum_{i<s<j} x_{i s} \otimes x_{s j} \quad \text { for all } 1 \leq i, j \leq n .
$$

In the following proposition, let $E=\left(e_{i}\right), F=\left(f_{i}\right)$ and $G=\left(g_{i}\right)$ be three arbitrary vectors in $k^{n-1}$. For $1 \leq i<j \leq n$, define

$$
a_{i j}=g_{i}+g_{i+1}+\cdots+g_{j-1}
$$

It follows from the definition that $a_{i s}+a_{s j}=a_{i j}$ for all $1 \leq i<s<j \leq n$.

EXAMPLE 5.4. Let $n \geq 3$. Then the following anti-commutative $k$-bilinear map [ , ] : $U_{n}^{\otimes 2} \rightarrow U_{n}$ defines a Lie algebra structure on $U_{n}$ such that $\left(U_{n},[],\right)$ is a coassociative 
Lie algebra.

$$
\begin{array}{rlrl}
{\left[x_{1 n}, x_{1 n}\right]} & =0, & \\
{\left[x_{s t}, x_{i j}\right]} & =0 & \text { if }(s, t) \neq(1, n) \text { and }(i, j) \neq(1, n), \\
{\left[x_{1 n}, x_{i j}\right]} & =a_{i j} x_{i j} & \text { if }(i, j) \neq(1, n),(1, n-1),(2, n), \\
{\left[x_{1 n}, x_{1 n-1}\right]} & =a_{1 n-1} x_{1 n-1}+\sum_{i=1}^{n-1} e_{i} x_{i i+1}, \\
{\left[x_{1 n}, x_{2 n}\right]} & =a_{2 n} x_{2 n}+\sum_{i=1}^{n-1} f_{i} x_{i i+1} .
\end{array}
$$

Proof. First we prove that $\left(U_{n},[],\right)$ is a Lie algebra. Let $K=\bigoplus_{(i, j) \neq(1, n)} k x_{i j} \subset L$. By definition, we have $[K, K]=0$ and $[L, K]=[K, L] \subset K$. Since we define $[$, ] to be anti-commutative, it suffices to show the Jacobi identity

$$
[a,[b, c]]=[b,[a, c]]+[[a, b], c]
$$

for all $a, b, c \in U_{n}$. If $a, b, c \in K$, then the Jacobi identity is trivially true. If $a=b=x_{1 n}$ and $c \in K$, the Jacobi identity is also true since it is true for all $a=b$. The Jacobi identity is stable under permutation and thus the remaining case to consider is when $a=x_{1 n}$ and $b, c \in K$. In this case,

$$
\begin{gathered}
{[a,[b, c]]=[a, 0]=0,} \\
{[b,[a, c]]+[[a, b], c] \in[K, K]+[K, K]=\{0\} .}
\end{gathered}
$$

Hence, the Jacobi identity holds and $\left(U_{n},[],\right)$ is a Lie algebra.

To prove $\left(U_{n},[],\right)$ is a coassociative Lie algebra, we need to verify (E2.1.1). By linearity, it suffices to check (E2.1.1) for cases listed in (E5.4.1)-(E5.4.5).

Case 1: If $(a, b)=\left(x_{1 n}, x_{1 n}\right),(\mathrm{E} 2.1 .1)$ is automatic (for any $\left.a=b\right)$.

Case 2: Suppose that $(a, b)=\left(x_{s t}, x_{i j}\right)$ for $(s, t) \neq(1, n)$ and $(i, j) \neq(1, n)$. Since $\delta(L) \subset K \otimes K$ and $[K, K]=0$, both sides of (E2.1.1) are zero.

Case 3: Suppose that $(a, b)=\left(x_{1 n}, x_{i j}\right)$ for $(i, j) \neq(1, n)$. Using the fact $[K, K]=0$, we have

$$
\begin{aligned}
\text { LHS of }(\mathrm{E} 2.1 .1) & =\delta\left(\left[x_{1 n}, x_{i j}\right]\right)=a_{i j} \sum_{i<s<j} x_{i s} \otimes x_{s j}, \\
\operatorname{RHS} \text { of }(\mathrm{E} 2.1 .1) & =\left[x_{1 n} \otimes 1+1 \otimes x_{1 n}, \sum_{i<s<j} x_{i s} \otimes x_{s j}\right] \\
& =\left(a_{i s}+a_{s j}\right) \sum_{i<s<j} x_{i s} \otimes x_{s j}=a_{i j} \sum_{i<s<j} x_{i s} \otimes x_{s j} .
\end{aligned}
$$

Hence, (E2.1.1) holds. This takes care of cases in (E5.4.3)-(E5.4.5).

Combining all the above cases, we have checked (E2.1.1). Therefore, $\left(U_{n},[],\right)$ is a coassociative Lie algebra.

ACKNOWLEDGEMENTS. The authors thank Chelsea Walton for reading an earlier version of this paper and for her useful comments. We thank Milen Yakimov for suggesting the name 'coassociative Lie algebra' for the main object studied in this paper. 
The authors also thank the referee for his/her valuable comments and suggestions. Part of the research was completed when J. J. Zhang visited Fudan University in the Fall of 2009, in the Spring of 2010 and in the Summer of 2011. D.-G. Wang was supported by the National Natural Science Foundation of China (Nos. 10671016 and 11171183) and the Shandong Provincial Natural Science Foundation of China (No. ZR2011AM013). J. J. Zhang and G. Zhuang were supported by the US National Science Foundation (NSF Grant No. DMS 0855743).

\section{REFERENCES}

1. K. A. Brown and J. J. Zhang, Prime regular Hopf algebras of GK-dimension one, Proc. London Math. Soc. 101(3) (2010), 260-302.

2. J.-W. He, F. Van Oystaeyen and Y. Zhang, Co-commutative Calabi-Yau Hopf algebras and deformations, J. Algebra 324 (2010), 1921-1939.

3. J.-J. Koszul, Homologie et cohomologie des algébres de Lie, Bull. Soc. Math. France 78 (1950), 65-127.

4. G. R. Krause and T. H. Lenagan, Growth of algebras and Gelfand-Kirillov dimension, Revised ed, Graduate Studies in Mathematics, 22 (American Mathematical Society, Providence, RI, 2000).

5. D.-M. Lu, Q.-S. Wu and J. J. Zhang, Homological integral of Hopf algebras, Trans. Amer. Math. Soc. 359(10) (2007), 4945-4975.

6. S. Montgomery, Hopf Algebras and their actions on rings, CBMS Regional Conference Series in Mathematics, 82 (American Mathematical Society, Providence, RI, 1993).

7. D.-G. Wang, J. J. Zhang and G.-B. Zhuang, Classification of connected Hopf algebras of GK dimension four, Trans. Amer. Math. Soc. (to appear).

8. A. Yekutieli, The rigid dualizing complex of a universal enveloping algebra, J. Pure Appl. Algebra 150 (2000), 85-93.

9. G. Zhuang, Existence of Hopf subalgebras of GK-dimension two, J. Pure Appl. Algebra 215 (2011), 2912-2922.

10. G. Zhuang, Properties of pointed and connected Hopf algebras of finite GelfandKirillov dimension, J. London Math. Soc. 87(2) (2013), 877-898. 